\title{
Where are the hot ion lines in classical T Tauri stars formed?
}

\author{
H. M. Günther and J. H. M. M. Schmitt
}

\author{
Hamburger Sternwarte, Universität Hamburg, Gojenbergsweg 112, 21029 Hamburg, Germany \\ e-mail: moritz.guenther@hs .uni-hamburg.de
}

Received 14 September 2007 / Accepted 14 January 2008

\begin{abstract}
Context. Classical T Tauri stars (hereafter CTTS) show a plethora of in- and outflow signatures in a variety of wavelength bands. Aims. In order to constrain gas velocities and temperatures, we analyse the emission in the hot ion lines.

Methods. We use all available archival FUSE spectra of CTTS to measure the widths, fluxes and shifts of the detected hot ion lines and complement these data with $H S T / G H R S$ and HST/STIS data. We present theoretical estimates of the temperatures reached in possible emission models such as jets, winds, disks and accretion funnels and look for correlations with X-ray lines and absorption properties. Results. We find line shifts in the range from $-170 \mathrm{~km} \mathrm{~s}^{-1}$ to $+100 \mathrm{~km} \mathrm{~s}^{-1}$. Most linewidths exceed the stellar rotational broadening. Those CTTS with blue-shifted lines also show excess absorption in X-rays. CTTS can be distinguished from main sequence (hereafter MS) stars by their large ratio of the O VII to O VI luminosities.

Conclusions. No single emission mechanism can be found for all objects. The properties of those stars with blue-shifted lines are compatible with an origin in a shock-heated dust-depleted outflow.
\end{abstract}

Key words. stars: formation - stars: winds, outflows - ultraviolet: stars

\section{Introduction}

T Tauri stars (TTS) are young $(<10 \mathrm{Myr})$, low mass $\left(M_{*}<\right.$ $\left.3 M_{\odot}\right)$, pre-main sequence stars exhibiting strong $\mathrm{H} \alpha$ emission. The subclass of Classical TTS (CTTS) are accreting material from a surrounding disk. This disk is truncated close to the corotation radius by interaction with the magnetic field. At the inner rim, the material is ionised by the stellar radiation and loaded onto the field lines (Uchida \& Shibata 1984; Koenigl 1991) and accelerated to nearly free-fall velocity along an accretion funnel before hitting the stellar surface where an accretion shock forms. Angular momentum may be transferred from the star to the disk by magnetic torque (Shu et al. 1994), at the same time this process is expected to drive outflows possibly in the form of a disk wind or bipolar jets. Romanova et al. (2004) performed full magneto-hydrodynamic (MHD) calculations of the accretion geometry, while the accretion shock and its post-shock cooling zone were simulated by different authors (Calvet \& Gullbring 1998; Lamzin 1998; Günther et al. 2007).

Observational support for the scenario described above is found in data from different wavelength bands. The shocked material is heated up to temperatures in the MK range and should produce copious amounts of X-ray emission. Density-sensitive $\mathrm{X}$-ray line ratios strongly suggest a post-shock origin (Günther et al. 2007) of the cool parts of the plasma observed at X-ray wavelengths, and the cooling matter veils absorption lines in the optical and infrared (Calvet \& Gullbring 1998). The geometry of the pre-shock accretion funnel can be traced by $\mathrm{H} \alpha$ line profile analysis (Muzerolle et al. 2000). Undoubtedly CTTS also show outflows (a review is given by Bally et al. 2007), however, the precise origin and the physical driving mechanism(s) are uncertain. Theoretical models propose a variety of collimated stellar outflows and disk winds to remove angular momentum from the system (Shu et al. 1994; Matt et al. 2002; Gómez de Castro \& Ferro-Fontán 2005; Pudritz et al. 2007). The outflows can take the form of winds observed from radio to the UV (e.g. Alencar \& Basri 2000; Beristain et al. 2001; Lamzin et al. 2004; Dupree et al. 2005a; Edwards et al. 2006), or collimated, often bipolar jets, again observed at different wavelength bands (Rodriguez 1995; Coffey et al. 2004; Güdel et al. 2005). The dynamics of the surrounding gas have been successfully probed by UV spectroscopy with $H S T / G H R S, H S T / S T I S$ and FUSE, especially in the $\mathrm{H}_{2}$ lines, which show outflows and fluorescence on the hot disk surface (Ardila et al. 2002a,b; Herczeg et al. 2002, 2005, 2006). In the specific case of TW Hya, Dupree et al. (2005a) claim the existence of a warm/hot wind. Their conclusion assumes that the CIII and OVI lines have an intrinsic Gaussian shape, with the centroid matching the stellar rest-frame. The observed asymmetry of the CIII and OVI line-profiles can then be explained by the presence of a continuous and smoothly-accelerated hot wind absorbing the radiation in the blue wing of these particular lines. However, Johns-Krull \& Herczeg (2007) argue that this model is incompatible with HST/STIS observations, especially for the C IV $1550 \AA$ doublet. The existence of a hot wind in TW Hya, therefore, remains an open issue.

In this paper we analyse the shifts and widths of hot-ion spectral lines, particularly C III $977 \AA$ and the O VI doublet at $1032 \AA$ and $1038 \AA$, observed with FUSE. This complements earlier studies of all pre-main sequence stars observed with $I U E$ (Valenti et al. 2000), GHRS (Ardila et al. 2002a,b) and of the $\mathrm{H}_{2}$ emission in CTTS (Herczeg et al. 2006). In Sect. 2 we briefly summarise the properties of the sample stars, and in Sect. 3 we describe the observations and data that we use in our present analysis. In Sect. 4 the line profile analysis is presented, several possible formation regions for the observed properties are discussed in Sect. 5, and in Sect. 6 we summarise our results. 


\section{Stellar properties}

In this section we present the most important properties of our sample stars in Table 1 and provide a short summary of their main characteristics.

\subsection{RU Lupi}

RU Lupi is a strong accretor, heavily-veiled and strongly variable at different wavelengths (Stempels \& Piskunov 2002). Its period is uncertain. A wind can be studied using cool, metallic lines with a low first-ionisation potential, and in $\mathrm{H}_{2}$ as demonstrated by Herczeg et al. (2005). The line profiles of hot ion lines in the UV exhibit complex structures. Takami et al. (2001) show by spectro-astrometry that the $\mathrm{H} \alpha$ emission is significantly extended and compatible with a bipolar outflow. In Xrays, RU Lupi is observed to be a typical member of the CTTS class (Robrade \& Schmitt 2007); specifically, it shows a low $f / i$ ratio in the O VII triplet as well as a soft X-ray excess.

\subsection{T Tau}

$\mathrm{T}$ Tau is the defining member of the class of TTS. It is a hierarchical triple system, where the northern component $\mathrm{T}$ Tau $\mathrm{N}$ is optically visible, while the southern component T Tau S, contributing mainly in the infrared, is itself a binary (Koresko 2000). The $\mathrm{T}$ Tau system shows a very significant soft X-ray excess without the high densities seen in TW Hya (Güdel et al. 2007). Nevertheless, accretion and winds are detectable using data for other wavelengths (van Langevelde et al. 1994b,a; Dupree et al. 2005a), and Herczeg et al. (2006), for example, attribute observed fluorescent $\mathrm{H}_{2}$ emission to outflows. The first analysis of this FUSE data, completed by Wilkinson et al. (2002), provided the first discovery of extra-solar, $\mathrm{H}_{2}$ Werner-band emission.

\subsection{DF Tau}

DF Tau is a binary system consisting of two M stars, resolved for the first time by Chen et al. (1990). The orbital parameters are still uncertain after several revisions (Schaefer et al. 2006) and the distance of the system is still a matter of debate. The most reasonable estimate is provided by Bertout \& Genova (2006), who showed DF Tau to be a member of the Taurus-Aurigae moving group $(\approx 140 \mathrm{pc})$, in spite of its low HIPPARCOS parallax of $39 \pm 7$ pc. Lamzin et al. (2001) present HST/STIS and IUE spectra showing accretion and wind signatures and attribute DF Tau's erratic photometric variability to unsteady accretion. DF Tau is one of few Doppler-mapped CTTS (Unruh et al. 1998). Herczeg et al. (2006) demonstrated that fluorescent $\mathrm{H}_{2}$ emission found in FUSE observations is consistent with models of a warm disk surface. X-ray observations for DF Tau are discussed in Sect. 3.3.

\subsection{V4046 Sgr}

Sallmen et al. (2000) present the FUSE spectrum of V4046 Sgr. V4046 Sgr is a close binary system with separation of $\approx 9 R_{\odot}$ (Quast et al. 2000). Both of the components are almost identical CTTS, which are accreting matter from a circumbinary disk. Chandra X-ray observations show line ratios indicative of high density plasma, which cannot be explained by coronal activity, but must be attributed to hot accretion shocks (Günther et al. 2006).

\subsection{TWA 5}

Sallmen et al. (2000) present the FUSE spectrum of TWA 5. TWA 5 is a hierarchical multiple system, the main component being a M 1.5 dwarf. On the one hand, TWA 5 has no significant infrared excess indicating the absence of a disk (Metchev et al. 2004; Weinberger et al. 2004; Uchida et al. 2004); also, X-ray observations by Argiroffi et al. (2005) suggest that the emission originates in a low-density region, not in an accretion shock. On the other hand, TWA 5 has an $\mathrm{H} \alpha$ equivalent width of $13.5 \AA$ and signatures of outflows (Mohanty et al. 2003), therefore we regard it as a transition object.

\subsection{GM Aur}

GM Aur is one of the most popular systems to observe stellar disk constitution and chemistry (e.g. Bergin et al. 2004; Hueso \& Guillot 2005; Salyk et al. 2007). Numerous observations in the radio use the disk of GM Aur to analyse the distribution and evolution of dust grains in the disk (e.g. Rodmann et al. 2006; Schegerer et al. 2006). Based on an optically-thin inner disk surrounded by an optically-thick outer region, Najita et al. (2007) classify GM Aur as a transition object. X-ray observations for GM Aur are discussed in Sect. 3.3.

\subsection{TW Hya}

Because of its proximity and the absence of a surrounding dark molecular cloud, TW Hya is a key system for the study of CTTS. Although relatively old by comparison $(\approx 10 \mathrm{Myr})$, it is still actively accreting from a surrounding disk. Eisner et al. (2006) interferometrically resolve its disk and Herczeg et al. (2004) constrain the inner disk temperature to about $2500 \mathrm{~K}$ using $\mathrm{H}_{2}$ fluorescence in HST/STIS and FUSE data. Observations with STIS and FUSE show, in addition to $\mathrm{H}_{2}$, atomic emission lines with notably asymmetric shapes. The analytical models expect a cold wind to be driven by magnetic fields from the disk (Shu et al. 1994), and this can be traced observationally from single or double ionised ions (Lamzin et al. 2004). TW Hya has been extensively observed in X-rays, which revealed a comparatively cool and dense emission region interpreted as signature of an accretion hot spot (Kastner et al. 2002; Stelzer \& Schmitt 2004). Our own theoretical modelling supports this view (Günther et al. 2007).

\section{Observations}

\subsection{FUSE data}

In order to perform a systematic study of the FUV properties of CTTS, we retrieved all available FUSE observations of TTS from the archive. For a comparison with "normal" stars, we selected two main sequence stars, the rapidly-rotating $\mathrm{K}$ star $\mathrm{AB}$ Dor and the solar analog $\alpha$ Cen A. Table 2 lists the observation logs; all targets except $\alpha$ Cen A are observed using the LWRS aperture with $30^{\prime \prime} \times 30^{\prime \prime}$ field of view. We reduced the data with CalFUSE v3.2.0 (Dixon et al. 2007), performing extractions of the complete data set and night-only time intervals to check for airglow contamination. With the exception of GM Aur, the spectral regions of interest are not affected by airglow. To coadd the individual exposures, we used the CalFUSE get_shift task and applied two different methods: both methods involved cross-correlation, the first using a stellar emission line (O VI $1032 \AA$ on the $1 \mathrm{aLiF}$ detector and C III $977 \AA$ on the 
Table 1. Stellar sample properties, $N_{\mathrm{H}}$ lists only values derived from X-ray observations.

\begin{tabular}{lccccccc}
\hline \hline Star & Spectral type & $\begin{array}{c}d \\
{[\mathrm{pc}]}\end{array}$ & $\begin{array}{c}v_{\text {rad }} \\
{\left[\mathrm{km} \mathrm{s}^{-1}\right]}\end{array}$ & $\begin{array}{c}v \sin i \\
{\left[\mathrm{~km} \mathrm{~s}^{-1}\right]}\end{array}$ & $\begin{array}{c}i \\
{\left[{ }^{\circ}\right]}\end{array}$ & $\begin{array}{c}N_{\mathrm{H}} \\
{\left[10^{21} \mathrm{~cm}^{-2}\right]}\end{array}$ & $\begin{array}{c}A_{\mathrm{V}} \\
{[\mathrm{mag}]}\end{array}$ \\
\hline RU Lup & G5V & 140 & $-1.9 \pm 0.02(17)$ & $9.1 \pm 0.9(17)$ & $24(19)$ & $1.8(15)$ & $0.07(9)$ \\
T Tau & G5V & 140 & $18(9)$ & $20 \pm 5(9)$ & $15(9)$ & $5(5)$ & $0.3(9)$ \\
DF Tau & M0-2 & 140 & $21.6 \pm 9.2(4)$ & $15.8 \pm 1.0(7)$ & $>60(21)$ & $3-8$ & $0.5(9)$ \\
V4046 Sgr & K5 & 83 & $-6.94(12)$ & $14.9 \pm 0.9(18)$ & $35(18)$ & $<2(6)$ & $0.0(18)$ \\
TWA 5 & M3V & 57 & $14(20)$ & uncertain $(20)$ & n.a. & $0.3(2)$ & n.a. \\
GM Aur & K5V & 140 & $24(10)$ & $13(3)$ & $56 \pm 2(16)$ & $0.2-0.4$ & $0.31(11)$ \\
TW Hya & K8V & 57 & $12.5 \pm 0.5(1)$ & $5 \pm 2(1)$ & $7 \pm 1(13)$ & $0.35(14)$ & $0.0(8)$ \\
\hline
\end{tabular}

References: (1) Alencar \& Batalha (2002); (2) Argiroffi et al. (2005); (3) Basri \& Batalha (1990); (4) Bouvier et al. (1986); (5) Güdel et al. (2007); (6) new fits to the data from Günther et al. (2006); (7) Hartmann \& Stauffer (1989); (8) Herczeg et al. (2004); (9) Herczeg et al. (2006); (10) Joy (1949); (11) Muzerolle et al. (1998) (12) Quast et al. (2000); (13) Qi et al. (2004); (14) Robrade \& Schmitt (2006); (15) Robrade \& Schmitt (2007); (16) Simon et al. (2000); (17) Stempels \& Piskunov (2002); (18) Stempels \& Gahm (2004); (19) Stempels et al. (2007); (20) Torres et al. (2003); (21) Unruh et al. (1998).

$2 \mathrm{bSiC}$ detector), and the second method using a strong airglow line $(\mathrm{H} \operatorname{Ly} \beta$ and $\mathrm{H} \operatorname{Ly} \gamma)$. Airglow lines completely cover the aperture and cannot therefore be used to judge centering of a target. We studied all stellar spectra with $\mathrm{O}$ VI and $\mathrm{C}$ III lines sufficiently strong for cross-correlation, and found no significant differences between the line profiles derived using both methods. Using airglow lines does, however, at times enable $\mathrm{H}_{2}$ emission to be detected in the $1 \mathrm{bLiF}$ channel. This is in contrast to the less accurate cross-correlation achieved using low $\mathrm{S} / \mathrm{N}$ stellar lines.

It seems that in our observations the targets did not drift through the apertures. Therefore we shift all airglow $\mathrm{H}$ Ly $\beta$ lines to match the laboratory wavelength. As the targets may be positioned differently in aperture we perform the following check: Herczeg et al. (2002) discovered molecular hydrogen at rest compared to the star, so we identify $\mathrm{H}_{2}$ lines in the $1 \mathrm{bLiF}$ channel in TW Hya (the shift between channels a and $b$ on the same chip is smaller than $8 \mathrm{~km} \mathrm{~s}^{-1}$, Dixon et al. 2007); the shift of our $\mathrm{H}_{2}$ lines should now match the radial velocity of TW Hya. This fixes our wavelength scale and from here on we use TW Hya as our reference spectrum. To avoid the need for a spectral identification, we search the $1 \mathrm{bLiF}$ channel of the other observations for strong features, which correlate with TW Hya. Only lines with the same strengths in full and night-only extractions are considered here. We also check that no airglow feature is expected at that position according to Feldman et al. (2001). The line widths we find are as small as expected (Herczeg et al. 2006), lending further credibility to the coaddition of single exposures using an airglow line. In addition, we cross-correlate larger regions of the spectra without strong airglow features and find that the required shifts are in agreement with the analysis of single lines. For RU Lup, T Tau and DF Tau we correct for the velocity of the $\mathrm{H}_{2}$ outflow relative to the star from Herczeg et al. (2006). Taking into account the stellar radial velocities, the lines shifts confirm the shifts adopted above from the H Ly $\beta$ lines. For V4046 Sgr, we do not have a HST/STIS measurement of $\mathrm{H}_{2}$ lines, and for TWA 5 and GM Aur no suitable features in the 1 bLiF channel were found. However, given the success of the wavelength calibration for other stars, we are quite confident that it is reliable also in these cases. We estimate the total wavelength error in the $1 \mathrm{a} / \mathrm{bLiF}$ channels to be $20 \mathrm{~km} \mathrm{~s}^{-1}$. We then correlate the $2 \mathrm{bSiC}$ channel with the $1 \mathrm{aLiF}$ channel using the stellar O VI $1032 \AA$ line. Unfortunately the signal-to-noise ratio (hereafter $\mathrm{SNR}$ ) in $2 \mathrm{bSiC}$ is much lower than in $1 \mathrm{aLiF}$ and for $\mathrm{T}$ Tau, DF Tau and GM Aur we have to use the $\mathrm{H} \mathrm{Ly} \beta$ airglow line. Because the FUSE channels are not perfectly aligned, the position of the target in the various channels might be different;
Table 2. FUSE observations.

\begin{tabular}{|c|c|c|c|}
\hline Target & $\overline{\text { Date }}$ & $\begin{array}{c}\text { Exposure time } \\
{[\mathrm{ks}]}\end{array}$ & $\overline{\text { Data ID }}$ \\
\hline RU Lup & $2001-08-28$ & 24 & A1090202 \\
\hline T Tau & 2001-01-15 & 21 & P1630101 \\
\hline DF Tau & 2000-09-13 & 26 & A1090101 \\
\hline V4046 Sgr & $2000-05-18$ & 16 & P1920202 \\
\hline TWA 5 & $2000-05-15$ & 15 & P1920101 \\
\hline GM Aur & 2004-03-03 & 32 & D0810101 \\
\hline GM Aur & $2004-10-23$ & 34 & D0810102 \\
\hline TW Hya & $2000-06-03$ & 2 & P1860101 \\
\hline TW Hya & $2003-02-20$ & 15 & C0670101 \\
\hline TW Hya & $2003-02-21$ & 15 & C0670102 \\
\hline AB Dor & 1999-10-20 & 22 & X0250201 \\
\hline AB Dor & $1999-12-14$ & 24 & X0250203 \\
\hline AB Dor & $2003-12-26$ & 102 & D1260101 \\
\hline$\alpha{\text { Cen } \mathrm{A}^{1}}^{1}$ & $2001-06-25$ & 15 & P1042601 \\
\hline 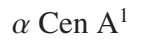 & 2006-05-05 & 13 & G0810102 \\
\hline
\end{tabular}

Notes: (1) MDRS aperture.

the wavelength calibration below $1000 \AA$ for these three stars is therefore uncertain.

For data of low SNR CalFUSE sometimes overcorrects the background, leading to negative flux intensity values; we therefore always separately fit the background in line-free regions, close to the line of interest.

\subsection{HST data}

Four of our sample stars (RU Lup, T Tau, DF Tau and TW Hya) have been observed with gratings onboard of the HST. RU Lup, T Tau and DF Tau were observed with the GHRS; these observations are presented by Ardila et al. (2002a) and Lamzin et al. (2001) and we adopt the linewidths and shifts measured by these authors. The STIS observations of RU Lup were carefully analysed by Herczeg et al. (2005) and those of TW Hya by Herczeg et al. (2002) and Johns-Krull \& Herczeg (2007). We use the STIS spectra of T Tau and DF Tau from CoolCAT (Ayres 2005), which is a database of reduced and calibrated UV spectra taken with STIS for cool stars. These spectra all belong to the HST program 8157 and were taken in the year 2000. The observations of TW Hya are not contained in CoolCAT, because they were taken in a non-standard setup. For a consistency check, we take $\mathrm{T}$ Tau, and find that the spectra presented there agree with the previously published results in Herczeg et al. (2005). 
Table 3. ROSAT observations.

\begin{tabular}{lccc}
\hline \hline Target & Date & $\begin{array}{c}\text { Exposure time } \\
{[\mathrm{ks}]}\end{array}$ & Obs ID \\
\hline DF Tau & $1993-08-93$ & 10 & WG201533P.N1 \\
GM Aur & $1992-09-22$ & 1.3 & WG201278P.N1 \\
GM Aur & $1993-03-04$ & 4 & WG201278P-1.N1 \\
\hline
\end{tabular}

\subsection{X-ray data}

Most of our sample stars have been observed at X-ray wavelengths with Chandra, XMM-Newton or both, and we obtained these data from the literature. For DF Tau and GM Aur, there are no X-ray spectra available in the literature, however, both DF Tau and GM Aur have been observed with ROSAT/PSPC pointings. We therefore retrieved the ROSAT X-ray data from the archive in order to enlarge our sample for comparisons of optical and X-ray absorption. In Table 3, we provide information on the data used. The ROSAT data were reduced using EXSAS version $030 c t$. Source photons were extracted from a circle with a radius of 2.5 arcmin to avoid contamination from nearby sources. The background was taken from a region close to the target without detected sources with a radius three times larger. We measured fluxes of $0.013 \pm 0.002 \mathrm{cts} \mathrm{s}^{-1}$ for DF Tau and $0.021 \pm 0.003 \mathrm{cts} \mathrm{s}^{-1}$ for GM Aur. Using MIDAS/EXSAS, we fitted Raymond-Smith and Mewe-Kaastra models assuming cold absorption. The ROSAT data are of low SNR. We therefore tried to use different data binnings and compared our final results. We provide a range of possible absorption columndensities in Table 1, because, as often found, observationally a large amount of cool plasma and small interstellar absorption lead to very similar signatures. To test the reliability of our procedure, we fit the ROSAT data for AB Aur, which is located in the same field as GM Aur. We measure an absorption columndenstiy $N_{\mathrm{H}}$ of $2-3 \times 10^{20} \mathrm{~cm}^{-2}$, which is approximately half of the measurement derived using XMM-Newton data presented by Telleschi et al. (2007). We therefore conclude that, taking into account systematic errors, the ROSAT-derived absorption columns are consistent with those derived using XMM-Newton data.

\section{Results}

\subsection{FUV lines}

In Fig. 1, we present the $\mathrm{C}$ III line profiles at $977 \AA$ and both components of the O VI doublet at $1032 \AA$ and $1038 \AA$ for our targeted stars. Each spectral line is fitted with a Gaussian profile and shown normalised with respect to the fitted peak value. The best-fit parameters for line flux, shift of the Gaussian-line centre, and its full width at half maximum (FWHM), ordered by the observed shift in the O VI $1032 \AA$ line, are given in Table 4, where the shift is corrected for the radial velocity of the star. The statistical errors on the shift and the FWHM given in the table are typically comparable to our systematic calibration uncertainties. The systematic errors on the fluxes are of the order $10-20 \%$. The O VI doublet line at $1032 \AA$ is detected in all sample stars and the $\mathrm{C}$ III line in all except GM Aur. In all cases, the line shifts measured in the $\mathrm{C} \mathrm{III} \mathrm{and} \mathrm{O}$ VI are consistent, arguing for related emission regions; only for $\mathrm{T}$ Tau is there a statistically significant difference, probably due to uncertainties in the wavelength calibration of $2 \mathrm{SiC}$ detector data, as discussed in Sect. 3.1.

In the case of RU Lupi, an absorption component is clearly visible in all three lines, which is also fitted with a Gaussian

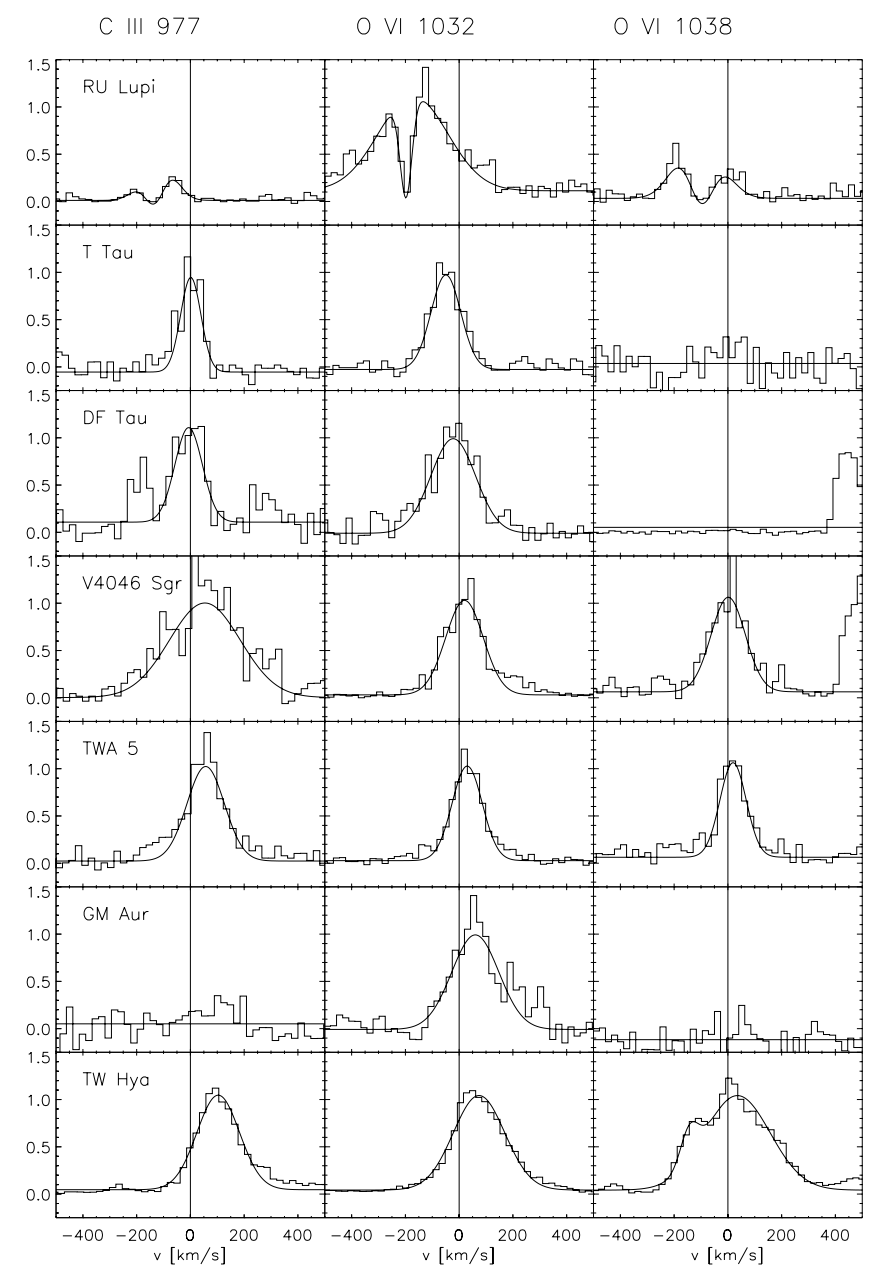

Fig. 1. Hot ion lines observed with FUSE in CTTS and best fit Gaussian profiles ordered by the shift in the O VI $1032 \AA$ line. All line profiles are normalised to their the Gaussian profile peak value and rebinned to instrumental resolution.

absorption line. We note that this absorption is not selfabsorption. Herczeg et al. (2005) identify the absorption in the O VI $1032 \AA$ as $\mathrm{H}_{2} 6-0 \mathrm{P}(3)$ shifted by $-23 \pm 5 \mathrm{~km} \mathrm{~s}^{-1}$ relative to the $\mathrm{H}_{2}$ rest wavelength, and the absorption in O VI $1038 \AA$ as C II. Using the package H2oolts (McCandliss 2003), we identify the absorption component in C III to be a superposition of the equally strong $\mathrm{H}_{2} 2-0 \mathrm{P}(5)$ and $11-0 \mathrm{R}(3)$ shifted by $\approx-10 \pm 15 \mathrm{~km} \mathrm{~s}^{-1}$ again relative to the $\mathrm{H}_{2}$ rest wavelength. Within the O VI $1038 \AA$ line of TW Hya, we fit the C II blend on the blue side with an additional Gaussian component. Unfortunately the data quality does not enable further blends and features to be identified. In the FUSE bandpass, numerous fluorescent Lyman and Werner band $\mathrm{H}_{2}$ lines are present, which are usually relatively sharp (Herczeg et al. 2006). The lines in Fig. 1 are welldescribed by one-peaked distributions, so the contribution of any individual blending line has to be small compared to the broad ion lines.

Atomic physics predicts that the $1032 \AA$ component of the O VI doublet should be twice as strong as the $1038 \AA$ component. We can confirm this prediction for the data of V4046 Sgr and TWA 5. In Table 4, significant deviations from the expected line ratio can be seen in RU Lup and TW Hya, where additional components complicate the fit. However, in the cases of T Tau, DF Tau and GM Aur the O VI $1038 \AA$ line is almost 
Table 4. FUSE best fit parameters, fluxes in $10^{-15} \mathrm{erg} \mathrm{cm}^{-2} \mathrm{~s}^{-1}$, width and shift in $\mathrm{km} \mathrm{s}^{-1}$, errors are statistical only.

\begin{tabular}{l|ccc|ccc|ccc|c}
\hline \hline & \multicolumn{3}{|c|}{ C III 977 $\AA$ O } & \multicolumn{3}{c|}{ O vi 1032 } & \multicolumn{3}{c|}{ O vi 1038 } & C III 1175 \\
Star & Flux & Shift & $F W H M$ & Flux & Shift & $F W H M$ & Flux & Shift & $F W H M$ & Flux \\
\hline RU Lup & $70 \pm 60$ & $-120 \pm 10$ & $120 \pm 20$ & $24 \pm 6$ & $-170 \pm 20$ & $300 \pm 60$ & $15 \pm 8$ & $-100 \pm 20$ & $170 \pm 30$ & $20 \pm 2$ \\
- Absorption & $-60 \pm 40$ & $-130 \pm 10$ & $100 \pm 20$ & $-4 \pm 0$ & $-200 \pm 6$ & $50 \pm 20$ & $-10 \pm 2$ & $-100 \pm 10$ & $120 \pm 30$ & $\ldots$ \\
T Tau & $10 \pm 4$ & $1 \pm 13$ & $90 \pm 30$ & $8 \pm 2$ & $-50 \pm 10$ & $130 \pm 30$ & n.a. & n.a. & n.a. & $18 \pm 2$ \\
DF Tau & $17 \pm 6$ & $-6 \pm 12$ & $120 \pm 30$ & $11 \pm 3$ & $-22 \pm 16$ & $200 \pm 40$ & n.a. & n.a. & n.a. & $44 \pm 2$ \\
V4046 Sgr & $60 \pm 20$ & $50 \pm 20$ & $310 \pm 70$ & $37 \pm 6$ & $19 \pm 8$ & $160 \pm 20$ & $16 \pm 4$ & $1 \pm 12$ & $150 \pm 30$ & $50 \pm 3$ \\
TWA 5 & $30 \pm 10$ & $60 \pm 20$ & $160 \pm 40$ & $30 \pm 5$ & $30 \pm 7$ & $130 \pm 20$ & $14 \pm 3$ & $19 \pm 9$ & $110 \pm 20$ & $25 \pm 2$ \\
GM Aur & n.a. & n.a. & n.a. & $5 \pm 1$ & $60 \pm 20$ & $210 \pm 60$ & n.a. & n.a. & n.a. & $24 \pm 2$ \\
TW Hya & $200 \pm 25$ & $100 \pm 6$ & $190 \pm 20$ & $220 \pm 20$ & $74 \pm 5$ & $220 \pm 15$ & $100 \pm 15$ & $35 \pm 15$ & $290 \pm 40$ & $310 \pm 4$ \\
- Blend & $\ldots$ & $\ldots$ & $\ldots$ & $\ldots$ & $\ldots$ & $\ldots$ & $9 \pm 9$ & $-140 \pm 14$ & $80 \pm 40$ & $\ldots$ \\
\hline
\end{tabular}
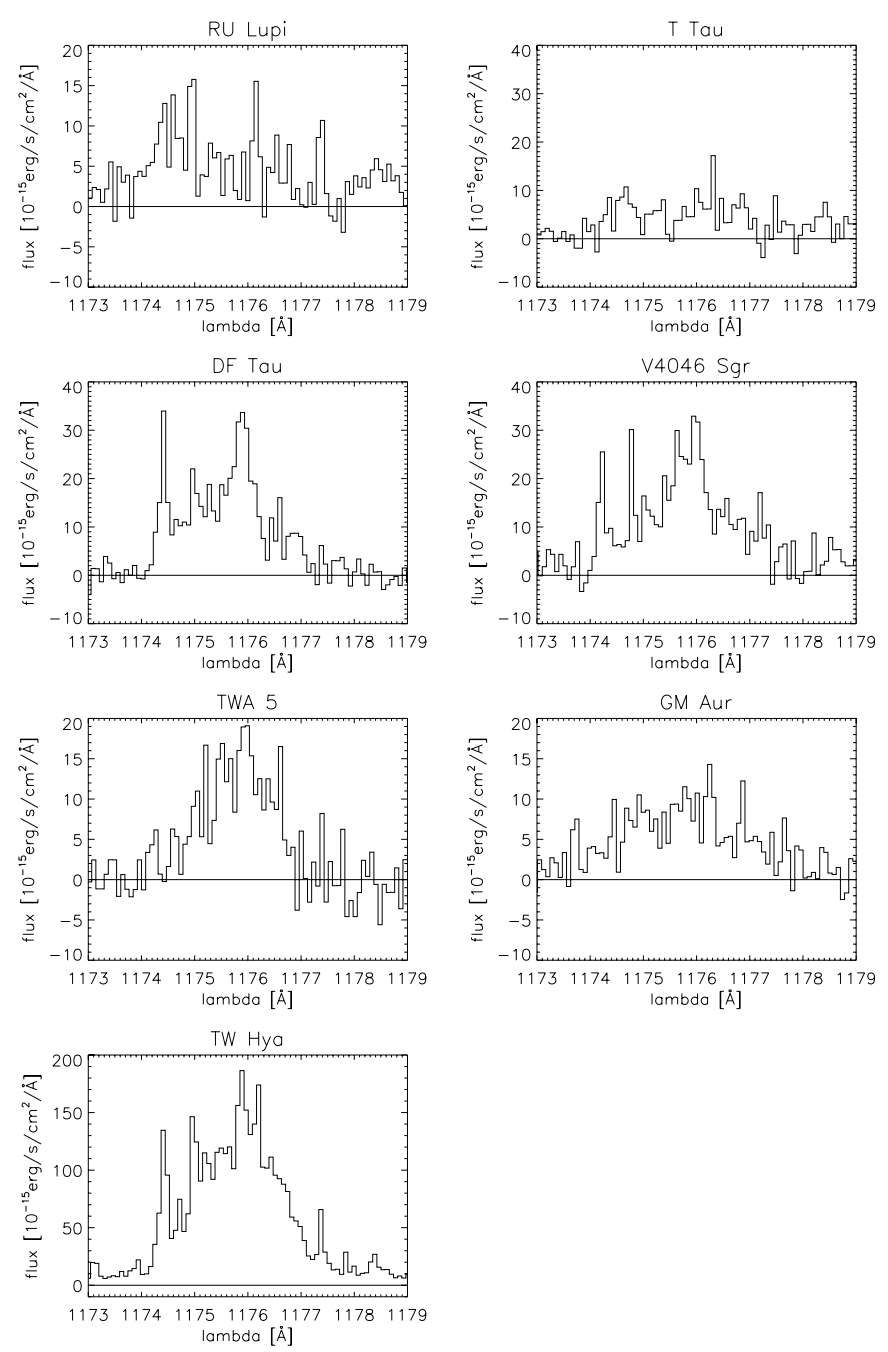

Fig. 2. The C III multiplet around $1175 \AA$ rebinned to instrumental resolution.

completely absent, because of absorption by larger molecular hydrogen columns (see Table 1) in the lines $\mathrm{H}_{2}$ 5-0 $\mathrm{R}(1)$ at 1037.1 $\AA$ and 5-0 P(1) at 1038.2 ̊. RU Lup and T Tau, both CTTS with blueshifted emission, are found to have the largest reddening and absorption column density.

In Fig. 2, we present the FUSE observations of the C III multiplet at wavelength $1175 \AA$. Unfortunately the SNR of the data is too low to be able to resolve the $\mathrm{H} 2$ lines, and to fit the individual line components. This analysis would have enabled density diagnostics to be measured. For RU Lup and T Tau in fact, the multiplet is barely detectable. Therefore a detailed Gaussian function

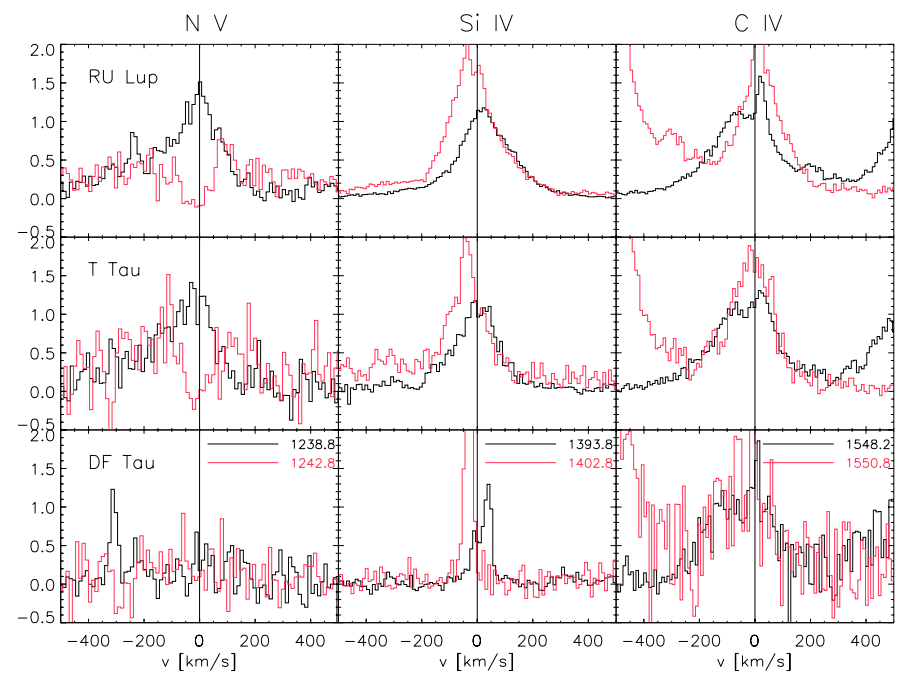

Fig. 3. Hot ion lines in the STIS spectra. The relative flux of the red doublet member is multiplied with a factor of 2 (red/grey lines).

fit yields no useful information, and in Table 4 we present only the total flux and its statistical error. The dereddened ratio of the fluxes in the C III $977 \AA$ line and the $1175 \AA$ multiplet is, in principle, density-sensitive in the range $8<\log n<11$ according to the CHIANTI database (Dere et al. 1998; Landi et al. 2006). However, apart from RU Lup the observed values are far below the predicted ratios. Redfield et al. (2002) have analysed this line ratio in FUSE observations of late-type dwarf stars, and Dupree et al. (2005b) for luminous cool stars. Both authors conclude that the C III $977 \AA$ line is optically-thick in the transition region and that in addition there is strong interstellar line absorption.

The STIS data from CoolCAT is shown close to the hot ionic doublets N V $1240 \AA$, Si IV $1400 \AA$ and C V $1550 \AA$ in Fig. 3 . In each case, the expected intensity ratio is two to one. To facilitate the comparison of the line profiles we show the flux of the red doublet member increased by a factor of two. In the absence of blending and interstellar absorption, both lines should show a perfect match. The red member of the N V doublet at $1242.8 \AA$ is totally absorbed by an interstellar N I line, so we do not attempt to fit the line profile. For the other lines, the results of a fit with a single Gaussian component are shown in Table 5. In DF Tau there is no overlap between the two lines shown in the middle panel of Fig. 3. Therefore they cannot be doublet members, but have to be other lines, likely $\mathrm{H}_{2}$. These lines also distort the red member of this doublet in the other two stars, leading to a shifted centre in the Gaussian fit. The large errors result from generally non-Gaussian line shapes. The Table also includes the GHRS data and the observations of TW Hya, resembling the 
Table 5. HST best fit parameters, for references see Sect. 3.2.

\begin{tabular}{|c|c|c|c|c|c|c|c|c|c|c|c|}
\hline \multirow[b]{2}{*}{ Star } & \multirow[b]{2}{*}{ Instr. } & \multicolumn{2}{|c|}{ N v $1239 \AA$} & \multicolumn{2}{|c|}{ Si IV $1394 \AA$} & \multicolumn{2}{|c|}{ Si IV $1403 \AA$} & \multicolumn{2}{|c|}{ C IV $1548 \AA$} & \multicolumn{2}{|c|}{ C IV $1551 \AA$} \\
\hline & & $\begin{array}{c}\text { Shift } \\
\mathrm{km} \mathrm{s}^{-1}\end{array}$ & $\begin{array}{l}F W H M \\
\mathrm{~km} \mathrm{~s}^{-1}\end{array}$ & $\begin{array}{c}\text { Shift } \\
\mathrm{km} \mathrm{s}^{-1}\end{array}$ & $\begin{array}{l}F W H M \\
\mathrm{~km} \mathrm{~s}^{-1}\end{array}$ & $\begin{array}{c}\text { Shift } \\
\mathrm{km} \mathrm{s}^{-1}\end{array}$ & $\begin{array}{l}F W H M \\
\mathrm{~km} \mathrm{~s}^{-1}\end{array}$ & $\begin{array}{c}\text { Shift } \\
\mathrm{km} \mathrm{s}^{-1}\end{array}$ & $\begin{array}{l}F W H M \\
\mathrm{~km} \mathrm{~s}^{-1}\end{array}$ & $\begin{array}{c}\text { Shift } \\
\mathrm{km} \mathrm{s}^{-1}\end{array}$ & $\begin{array}{l}F W H M \\
\mathrm{~km} \mathrm{~s}^{-1}\end{array}$ \\
\hline RU Lup & GHRS & & & $5 \pm 5$ & $270 \pm 10$ & $-31 \pm 6$ & $250 \pm 10$ & $-20 \pm 20$ & $340 \pm 40$ & $-10 \pm 10$ & $300 \pm 30$ \\
\hline RU Lup & STIS & $-15 \pm 25$ & $190 \pm 70$ & $26 \pm 20$ & $200 \pm 50$ & $-20 \pm 20$ & $180 \pm 50$ & $-25 \pm 25$ & $230 \pm 80$ & $15 \pm 18$ & $120 \pm 50$ \\
\hline T Tau & GHRS & & & $-15 \pm 7$ & $220 \pm 20$ & $-50 \pm 10$ & $220 \pm 30$ & $-25 \pm 6$ & $250 \pm 10$ & $0 \pm 6$ & $150 \pm 10$ \\
\hline T Tau & STIS & $-45 \pm 30$ & $220 \pm 80$ & $-1 \pm 20$ & $180 \pm 50$ & $-40 \pm 20$ & $130 \pm 50$ & $-25 \pm 20$ & $230 \pm 70$ & $-10 \pm 20$ & $150 \pm 50$ \\
\hline DF Tau & GHRS & $\ldots$ & & $\ldots$ & 300 & $\ldots$ & 300 & $10 \pm 10$ & $350 \pm 30$ & $10 \pm 10$ & $320 \pm 20$ \\
\hline DF Tau & STIS & $30 \pm 40$ & $187 \pm 85$ & 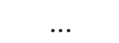 & 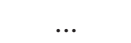 & . & ... & $-40 \pm 40$ & $230 \pm 100$ & $-50 \pm 40$ & $180 \pm 120$ \\
\hline TW Hya & STIS & redshifted & , see text & $42 \pm 3$ & $170 \pm 6$ & $9 \pm 3$ & $130 \pm 8$ & reds & hifted, non-C & aussian, se & text \\
\hline
\end{tabular}

FUSE data. These profiles are nearly triangular, rising sharply close to the rest wavelength and extending to about $400 \mathrm{~km} \mathrm{~s}^{-1}$ on the red side (see Fig. 7 in Herczeg et al. 2002). Of similar shape, although extending only to $200 \mathrm{~km} \mathrm{~s}^{-1}$ on the red side, is the C IV $1550 \AA$ doublet in DF Tau (Lamzin et al. 2001) in the GHRS observation. This data is fitted with multiple Gaussians by Ardila et al. (2002a). We give here the values for the dominant component only.

Despite the uncertainties in the profiles of individual lines, it is obvious from Tables 4 and 5 and Figs. 1 and 3, that FUV lines in CTTS cover a range of wavelength shifts from blueshifted to red-shifted velocities of the order of $-200 \mathrm{~km} \mathrm{~s}^{-1}$ out to $100 \mathrm{~km} \mathrm{~s}^{-1}$, and could therefore potentially originate in in- or outflows or both. RU Lup clearly shows blue-shifted emission, the same is likely the case for T Tau, because the O VI shift can be more accurately determined than the C III shift. DF Tau and V4046 Sgr are consistent with centred lines and TWA 5, GM Aur and TW Hya with red-shifted lines.

\subsection{Excess absorption}

In Fig. 4, we show the absorbing column densities as fitted to the $\mathrm{X}$-ray observations and optical $A_{\mathrm{V}}$ values. With a standard gasto-dust ratio, they should be related through the formula $N_{\mathrm{H}}=$ $A_{\mathrm{V}} \cdot 2 \times 10^{22} \mathrm{~cm}^{-2}$ (Savage \& Mathis 1979; but see Vuong et al. 2003 , for a compilation of other conversion factors in the literature, all roughly consistent with this value). The determination of $A_{\mathrm{V}}$ values is difficult for CTTS, where not only the spectral type, but also the veiling needs to be known to compute the intrinsic colours. This renders $A_{\mathrm{V}}$ values in the literature notoriously uncertain, especially if they are based only on photometric and not on spectroscopic information. We have tried to compile the best available estimates in Table 1. For AA Tau and possibly other CTTS, the circumstellar absorption and the veiling are timedependent. In Fig. 4, we show mean values for AA Tau from Schmitt \& Robrade (2007) and Bouvier et al. (1999). The $A_{\mathrm{V}}$ for $\mathrm{T}$ Tau is often given one magnitude larger than the value we use, but Walter et al. (2003) convincingly show that this is an overestimate. In the case of DF Tau, most values are between 0.21 (Kenyon \& Hartmann 1995) and 0.55 (Strom et al. 1989), with the exception of $1.9 \pm 0.6$ in Cohen \& Kuhi (1979); for RU Lup, all modern measurements agree on the low $A_{\mathrm{V}}$ value (Lamzin et al. 1996; Stempels \& Piskunov 2002; Herczeg et al. 2005). In addition to the TTS studied in this paper, we included data for the young stars BP Tau and SU Aur from Robrade \& Schmitt (2006) and Errico et al. (2001), for BP Tau, AB Aur and HD 163296 from Telleschi et al. (2007, see references therein) and for MP Mus from Argiroffi et al. (2007) and Mamajek et al. (2002). In the case of AA Tau, reddening and absorbing column are exceptionally large, because this object is seen almost edge-on.

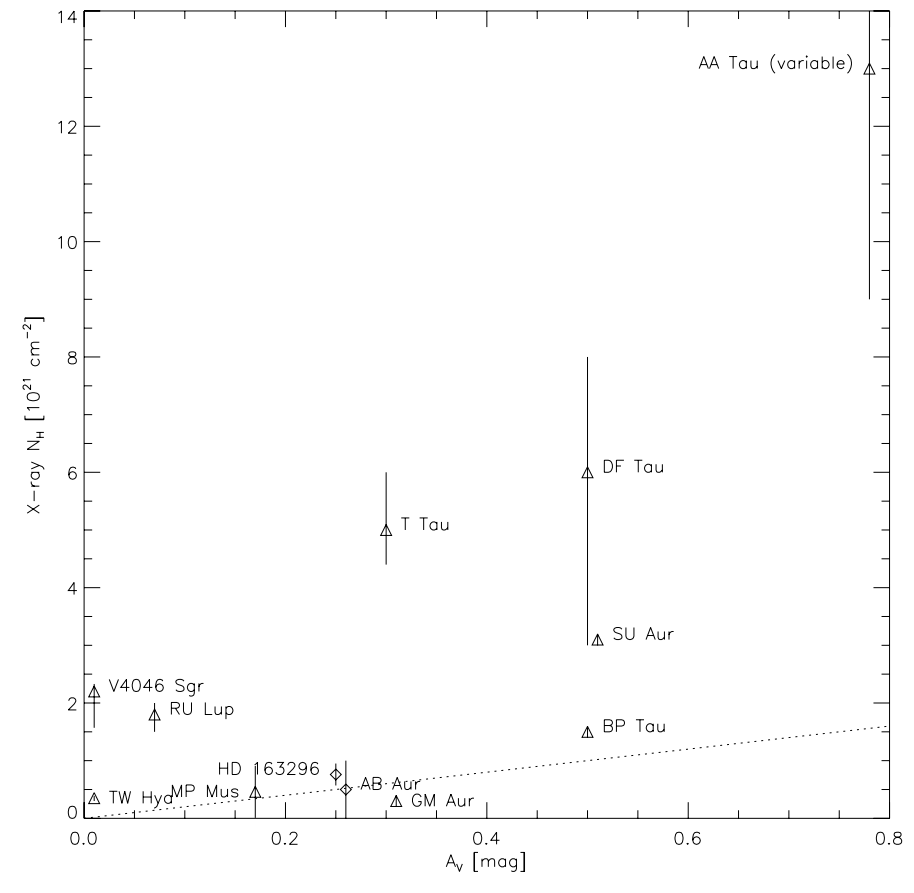

Fig. 4. Optical reddening and absorbing column density from X-rays. Error bars represent $90 \%$ confidence intervals on the $N_{\mathrm{H}}$ fit, except for DF Tau and GM Aur where we give the (tighter) ranges discussed in Sect. 3.3. The dotted line indicates the values predicted using $N_{\mathrm{H}}=$ $A_{\mathrm{V}} \cdot 2 \times 10^{22} \mathrm{~cm}^{-2}$.

AA Tau, DF Tau, T Tau, RU Lup and V4046 Sgr all show much larger X-ray absorbing columns than expected from the optical extinction, indicating large amounts of gas with a very small dust content compared to the interstellar medium. This effect is significantly larger than the uncertainties in the $A_{\mathrm{V}}$ and $N_{\mathrm{H}}$ values. In V4046 Sgr the relative uncertainties are higher compared to the other stars, because Chandra is not as sensitive as XMMNewton at soft X-ray energies. In RU Lup and, possibly, T Tau the hot ion lines exhibit significant blue shifts. Therefore, it may well be that the hot UV-emitting plasma provides the observed $\mathrm{X}$-ray excess absorption.

All $N_{\mathrm{H}}$ values provided are obtained by fitting a cold absorber model, implying that the elements contributing to the $\mathrm{X}$-ray absorption are not fully ionised. The most important elements are $\mathrm{O}, \mathrm{H}$, and $\mathrm{He}$; with increasing temperature and ionisation an even larger column density is necessary to provide the observed absorption. 


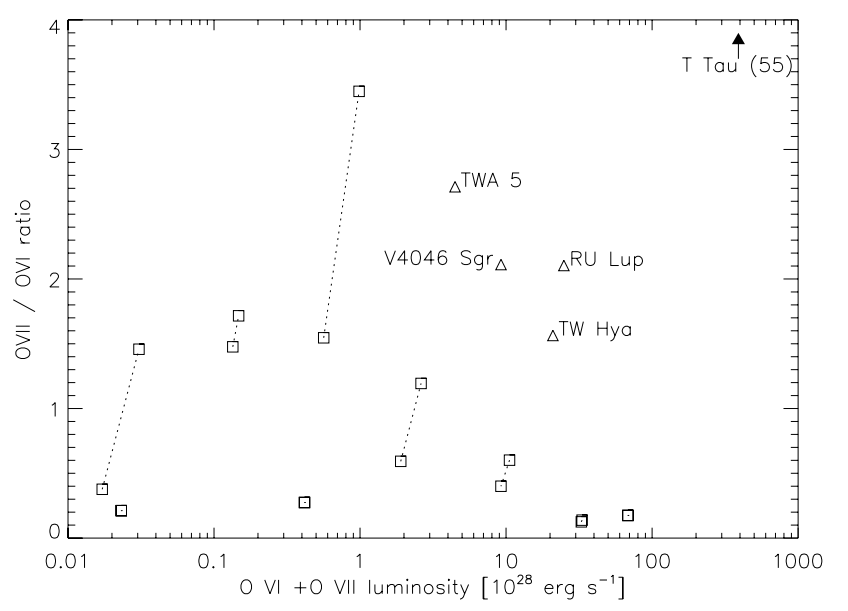

Fig. 5. Ratio of dereddened fluxes in the O VII triplet at $22 \AA$ to the O VI $1032 \AA$ line vs. total luminosity in those lines for CTTS (triangles) and other stars (squares). See text for data sources.

\subsection{Ratio of O VII to O VI luminosity}

X-ray data are available for all CTTS sample stars, most of which were observed using gratings. The resolution of these $\mathrm{X}$-ray lines is insufficient to determine velocities, and we therefore analyse only the measured line fluxes. In Fig. 5, we compare the fluxes of the O VI and O VII lines, where the X-ray data is taken from the same references as $\mathrm{N}_{\mathrm{H}}$ in Table 1 . The observed fluxes are dereddened (Cardelli et al. 1989) in the UV with a standard dust grain distribution assuming $R_{\mathrm{V}}=3.1$, and in the X-rays following the cold absorption model by BalucinskaChurch \& McCammon (1992). For comparison, some stars with "normal" coronal X-ray emission have been added (Ness et al. 2004), whose O VI emission is known (Redfield et al. 2002; Dupree et al. 2005b). Where Ness et al. (2004) provide measurements from both XMM-Newton and Chandra, we show two connected symbols to give an idea of the intrinsic variability of the sources. This by far dominates the observational uncertainties, which are approximately $10 \%$. As is clear from Fig. 5, the CTTS separate well out in the upper right corner of the plot, indicating an O VII excess compared to dwarf and giant cool stars. Robrade \& Schmitt (2007) and Güdel \& Telleschi (2007) show a very similar analysis using the O VIII/O VII ratio again with an $\mathrm{O}$ VII excess. These studies clearly show that the observed excess radiation of CTTS is confined to a relatively narrow temperature range about the formation of the He-like $\mathrm{O}$ VII triplet at 1-2 MK. We note that the CTTS T Tau has an unusually strong O VII excess, with a ratio close to 55 . Estimating the $\mathrm{H}_{2}$ column density to be roughly a quarter of the total neutral hydrogen absorbing column (Bloemen 1987), no more then about a third of the O VI flux in T Tau could be absorbed and the true blueshift cannot exceed $100 \mathrm{~km} \mathrm{~s}^{-1}$. Otherwise emission blueward of the absorbing $\mathrm{H}_{2} \mathrm{P}(3)(6-0)$ line at $1031.19 \AA$ would be observed in Fig. 1. Absorption in the $\mathrm{O}$ VI lines cannot therefore explain the extreme $\mathrm{O}$ VII to $\mathrm{O}$ VI ratio. A possible origin for the large emission measure at $1-2 \mathrm{MK}$ might be a small-density accretion shock with a large filling factor.

\section{Interpretation}

In the following we attempt to constrain the emission regions and set limits and constraints on possible models for the origin of the observed line shapes.

\subsection{Blue-shifted emission}

The detected emission of hot-ion lines in the FUSE data appears blue-shifted in RU Lupi and, probably, in T Tau. Both systems show small values of $v \sin i$ compared to zero-age main-sequence stars and their inclination is close to face-on (Table 1). However, TW Hya is a counter-example with small inclination and redshifted emission.

\subsubsection{Shocks in outflows}

We assume that an outflow exists, produced by either a disk or stellar wind. Blue-shifted emission could then be due to either internal shock fronts or to termination shocks of outflows running e.g. into an ISM cloud. For the case of RU Lupi, the measured centroid velocity is found to be $v_{\text {obs }} \approx 200 \mathrm{~km} \mathrm{~s}^{-1}$ away from the star, and the peak formation temperature of $\mathrm{O}$ VI is $300000 \mathrm{~K}$ (Mazzotta et al. 1998). If we assume that the O VI gas is heated by a strong shock wave, following Zel'Dovich \& Raizer (1967) we can infer that the shock front properties from the Rankine-Hugoniot jump conditions. The emission of a postshock cooling zone would be observed with velocity $v_{\text {obs }}$, which sets a lower boundary on the shock velocity $v_{\text {shock }}$ to

$4 v_{\text {obs }}=v_{\text {shock }} \geq 800 \mathrm{~km} \mathrm{~s}^{-1}$.

By using line shifts we measure only the line of sight velocity, but RU Lupi and T Tau are probably viewed close to poleon, thus we expect this value to be close to the true velocity. Typical jets reach up to $400 \mathrm{~km} \mathrm{~s}^{-1}$ (Eislöffel \& Mundt 1998), but Rodriguez (1995) report velocities of up to $1000 \mathrm{~km} \mathrm{~s}^{-1}$. The highest bulk velocities observed in the jet of the CTTS DG Tau are about $500 \mathrm{~km} \mathrm{~s}^{-1}$ (Lavalley-Fouquet et al. 2000). Using the strong shock formula the post-shock temperature $T_{\text {post }}$ can be obtained by

$$
\begin{aligned}
T_{\text {post }} & =\frac{\gamma-1}{(\gamma+1)^{2}} \frac{2 \mu m_{\mathrm{H}}}{k} v_{\text {shock }}^{2} \\
& =\frac{3 \mu m_{\mathrm{H}}}{k} v_{\mathrm{obs}}^{2},
\end{aligned}
$$

where $m_{\mathrm{H}}$ is the mass of an hydrogen atom, $\mu$ the dimensionless mean particle mass, $k$ is Boltzmann's constant and $\gamma$ is the adiabatic index. In the last step, we use $\gamma=5 / 3$ for an ideal gas and Eq. (1). The estimated post-shock temperatures of $\approx 2 \times 10^{6} \mathrm{~K}$ for RU Lup and $\approx 2 \times 10^{5} \mathrm{~K}$ for T Tau are large enough to form O VI and O VII. This seems plausible, because Güdel et al. (2005) present already spatially-resolved X-ray emission from a CTTS jet in DG Tau A. The shifts of the O VI lines observed in RU Lup and $\mathrm{T}$ Tau are larger than measured for the C III lines, agreeing with models of post-shock cooling. C III is formed at lower temperatures, when the post-shock flow has lost energy, cooled and slowed down.

The observed volume emission measures (in units of $10^{51} \mathrm{~cm}^{-3}$ ) for RU Lup, T Tau and DF Tau in the O VI $1032 \AA$ and $1038 \AA$ lines are 1.3, 1.4 and 4.7, assuming the temperature of maximum emissivity and dereddening of fluxes by Cardelli et al. (1989); for C III the corresponding numbers are 3.9, 1.5 and 8.0. Along the jet of the CTTS DG Tau pre-shock densities between $10^{3}$ and $10^{5} \mathrm{~cm}^{-3}$ are observed, with the higher values closer to the star (Lavalley-Fouquet et al. 2000). We now use the fit-formula from Hartigan et al. (1987) to estimate the cooling distance $d$ and we find $d \approx 3 \times 10^{16} \mathrm{~cm}$ for RU Lup with $v=800 \mathrm{~km} \mathrm{~s}^{-1}$ and $n=10^{4} \mathrm{~cm}^{-3}$. The diameter of the jet is then of the order $10^{13} \mathrm{~cm}$ and the column density of the cooling 


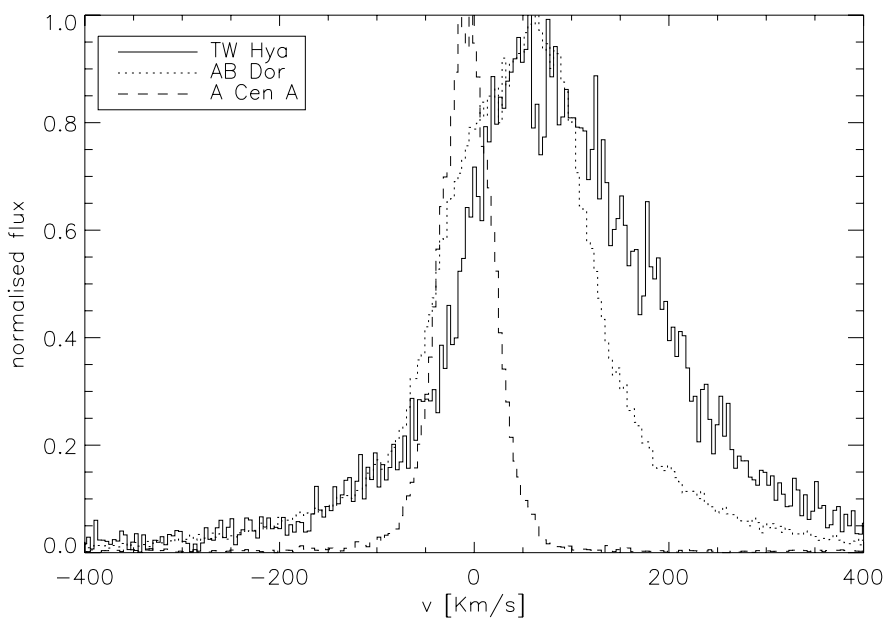

Fig. 6. Comparison of the O VI $1031.91 \AA$ line for three stars.

zone alone $N_{\mathrm{H}}=3 \times 10^{20} \mathrm{~cm}^{-2}$, adding to the absorbing column in the base of the jet, the circumstellar material further out and the interstellar column density. Therefore the large column densities observed for the stars with blue-shifted emission might be partly due to jet material. In T Tau and DF Tau the observed velocities are lower, which results in $d \approx 3 \times 10^{14} \mathrm{~cm}$. This is too small to explain the observed emission measures. In the jet emission picture this suggests jets inclined to the line of sight, so the velocities and therefore the cooling distances are underestimated. According to the data in Table 1, the inclination of $\mathrm{T}$ Tau is smaller than the inclination of RU Lup, but it is not clear if jets are always orientated perpendicular to the accretion disk. DF Tau has the largest inclination, so we expect to see the jet at a high inclination. This might explain the large emission measure despite the low observed shift, which is even consistent with centred emission.

It could thus well be that the additional absorption of the stellar X-ray emission is due to the hot gas from which the FUV lines originate.

\subsection{Anomalous line widths}

A comparison of rotational velocities listed in Table 1 with the line widths shown in Tables 4 and 5 shows that the observed lines are superrotationally-broadened despite the measurement uncertainties. This is not uncommon in late-type stars (Wood et al. 1997). To illustrate this issue further, in Fig. 6 we compare the O VI $1032 \AA$ line measured for TW Hya, the much more rapidly rotating $\mathrm{K}$ dwarf $\mathrm{AB}$ Dor with $v \sin i=100 \pm 5 \mathrm{~km} \mathrm{~s}^{-1}$ (Vilhu et al. 1987), and the slow-rotator $\alpha$ Cen with $v \sin i=$ $2.7 \pm 0.7 \mathrm{~km} \mathrm{~s}^{-1}$ (Saar \& Osten 1997) is in fact comparable to TW Hya. Ake et al. (2000) found significantly superrotationallybroadened coronal lines already in $\mathrm{AB}$ Dor and speculate that this might be due to microflare heating. The blue wing of TW Hya matches the data from AB Dor quite well, but the asymmetric profile from TW Hya is significantly different on the red side. At any rate, the O VI $1032 \AA$ is clearly far broader than the line from $\alpha$ Cen. In CTTS, redward line asymmetries are usually attributed to emission in the accretion process. We therefore consider if this interpretation is also valid for TW Hya.

\subsection{Magnetospheric infall}

\subsubsection{The basic model}

In the standard accretion scenario for CTTS, the disk is truncated at a few stellar radii and the temperatures on the disk surface are a few thousand $\mathrm{K}$ at most (Herczeg et al. 2004). Consequently, excluding additional energy deposition by magnetic star-disk interaction, the accreting material reaches the formation temperature of $\mathrm{O}$ VI for the first time shortly before it passes through the accretion shock. At this stage, the material on the opposite side of the star ought to be blocked from view and only the low velocity, low temperature zones of the accretion funnel, close to their origin on the disk, can be seen blue-shifted if they face the observer. Therefore hot ion lines can be observed only red-shifted from the funnel flow.

Using the CHIANTI 5.2 database (Dere et al. 1998; Landi et al. 2006) we have numerically modelled the accretion spot as described in detail by Günther et al. (2007) for TW Hya and for V4046 Sgr (Günther \& Schmitt 2007). We measure the infall velocities in the accretion funnel to be $525 \mathrm{~km} \mathrm{~s}^{-1}$, and the preshock densities around $10^{12} \mathrm{~cm}^{-3}$ for TW Hya and $2 \times 10^{11} \mathrm{~cm}^{-3}$ for V4046 Sgr. This matter passes through a shock on the stellar surface and is heated up to temperatures of $\approx 2 \times 10^{6} \mathrm{~K}$. X-ray emission is produced in the following cooling flow in the postshock zone. We explicitly calculate the ionisation and recombination rates, to be able to assess the ionisation state at each depth. Fitting this model to high-resolution X-ray data, we measure the abundances of the most important elements and determine mass accretion rates and filling factors between $0.1-0.4 \%$.

Our model explicitly assumes optically thin-radiation loss. Along the direction of infalling flow, most resonance lines are found to be optically-thick up to optical depths above 10 . The opacity is measured using a simple opacity calculation, and its precise value depends on both the infall density and elemental abundance. In the He-like triplets of Ne IX and O VII, observed in X-rays, the ratio of resonance line to intercombination plus forbidden line is expected to be close to unity from atomic physics. This is matched by observations, so we can infer that the resonance lines do not suffer much absorption. We note that the detailed accretion region geometry is unknown. In particular, the system geometry is probably not $1 \mathrm{D}$ and photons may well escape through the boundaries of the system rather than along the direction of flow. Unfortunately, this leads to considerable uncertainty in the computed flux. In our simulation however, emission is generated in the post-shock region to the same extent as observed for the OVI triplet, but the post-shock contribution to the CIII line is small. We caution that this conclusion is based on the assumption of very small and hot spots. Theoretical simulations of the infall geometry using either dipolar fields (Romanova et al. 2004) or more realistic geometries scaled from other stars (Gregory et al. 2006) suggest inhomogeneous spots with a distribution of infall velocities. In accretion zones with smaller infall velocities, the post-shock temperatures and hence the emitted X-ray fluxes are smaller. Such effects are not included in our models, but could contribute to the observed FUV flux. We are unable to reliably constrain fits of inhomogeneous spots due to insufficient diagnostics.

\subsubsection{Predicted UV line shifts}

For TW Hya and V4046 Sgr, where we modelled the accretion shock in detail, the largest possible post-shock speed is $125 \mathrm{~km} \mathrm{~s}^{-1}$, a quarter of the free-fall velocity. All emission 


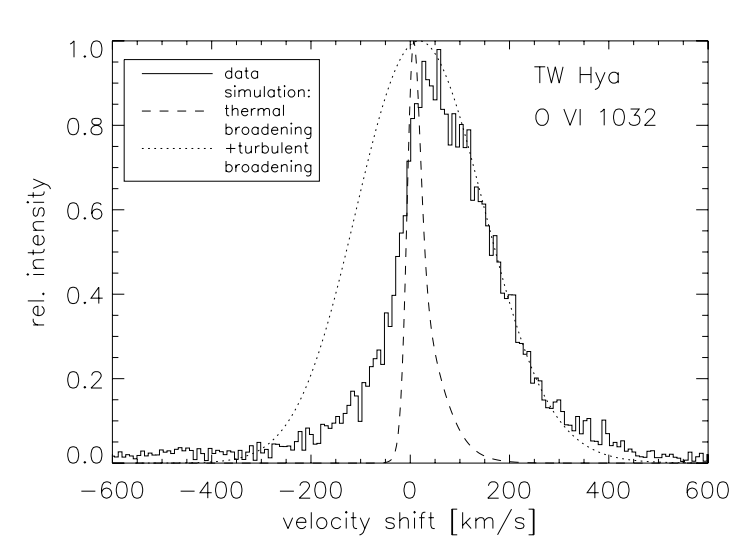

Fig. 7. O vi line at $1032 \AA$. Solid line: FUSE data, dashed line: simulation with purely thermal broadening, dotted line: simulation with additional turbulent broadening of $150 \mathrm{~km} \mathrm{~s}^{-1}$

redward of this value must originate in the pre-shock radiative precursor.

During the cooling behind the shock front, the velocity decreases further. The emission from $\mathrm{C}$ III and O VI peaks at very low velocities. For pre-shock velocities of $300 \mathrm{~km} \mathrm{~s}^{-1}$, O VI is formed immediately behind the accretion shock. Its velocity reaches a maximum value of $75 \mathrm{~km} \mathrm{~s}^{-1}$, and decreases as the cooling proceeds. Depending on the stellar inclination and the spot latitude, the projected velocity may be even smaller. The resulting line profile has its maximum close to $v=0 \mathrm{~km} \mathrm{~s}^{-1}$ with a broad wing on the red side. Observationally the $\mathrm{O}$ VI line at $1032 \AA$ is best suited for analysis, because the other member of the doublet at $1038 \AA$ is more strongly absorbed by $\mathrm{H}_{2}$ and possibly by C IV lines. The observed FWHM is about $200 \mathrm{~km} \mathrm{~s}^{-1}$ (Fig. 7). Adding turbulent broadening to our 1D simulation results in more symmetric line profiles, the red wing can be fitted with $v_{\text {turb }}=150 \mathrm{~km} \mathrm{~s}^{-1}$, but no blue wing is observed. In principle, this could be due to wind absorption, as suggested by Dupree et al. (2005a). In the case of TW Hya however, JohnsKrull \& Herczeg (2007) were able to demonstrate that a hot wind is incompatible with, for example, the observed C IV line profiles.

\subsubsection{Model extensions}

Depending on the accretion geometry, radiation from the postshock zone may be reprocessed in the pre-shock accretion flow. This will form a photoionised radiative precursor, sufficiently hot to emit in $\mathrm{C}$ III and even O VI lines. The emitting region however is located close to the shock, where the material is moving at close to free-fall speed, and emission is redshifted by about $500 \mathrm{~km} \mathrm{~s}^{-1}$. Lamzin (2003) performed detailed 3-level-atom calculations supporting the above considerations with numerical simulations. He found that the total emission should be observed as two distinct peaks, one close to free-fall velocity about $500 \mathrm{~km} \mathrm{~s}^{-1}$ and the other formed post-shock. Only if the line of sight is perpendicular to the direction of flow these two peaks can coincide, but close to the rest wavelength. For TW Hya, which is seen close to pole-on, this would require accretion in an equatorial layer.

Figure 7 shows that in principle a large turbulent broadening could explain the observed line width, but it requires turbulent velocities larger than the post-shock bulk velocity. Nevertheless other plasma processes such as magnetic waves may supply the required turbulence.

The line widths could be explained if the emission originates in the funnels well before entering the radiative precursor, in a region high above the stellar surface. A single feature corotating with the star would be observed moving through the line profile, as the star rotates, and a combination of many emission regions may result in a very broad line. In this case an efficient and currently unknown heating mechanism in the accretion funnels is needed to form the hot ions. The resolution of the current X-ray telescopes Chandra and XMM-Newton is insufficient to detect line broadening less than about $1000 \mathrm{~km} \mathrm{~s}^{-1}$ or line shifts less than a few $100 \mathrm{~km} \mathrm{~s}^{-1}$. We are therefore unable to compare our line-profile measurements with those for hotter ion lines, which are most likely accretion-dominated.

\subsection{Turbulence in boundary layers}

Turbulence is an obvious way to generate significant line widths. Turbulent flows convert bulk kinetic motion into heat and vorticity and a flow along a boundary layer would be an obvious origin of turbulence. If, alternatively, accretion proceeds in an equatorial boundary layer, the difference in velocity is given by the difference between the stellar rotation $v_{\text {rot }}$, and the velocity of material accreting close to the Keplerian velocity at the stellar surface $v_{\text {Kepler }}=\sqrt{\frac{G M_{*}}{R_{*}}} \approx 440 \mathrm{~km} \mathrm{~s}^{-1}$, where $G$ is the gravitational constant, and the stellar parameters radius $R_{*}$ and mass $M_{*}$ for the example of TW Hya are taken from Webb et al. (1999). The equatorial velocity can be estimated from $v_{\mathrm{eq}}=\frac{v \sin i}{\sin i} \approx 40 \mathrm{~km} \mathrm{~s}^{-1}$.

In order to estimate the dissipated energy, we use the turbulent Crocco-Busemann relation, which is valid for turbulent flow along a surface. We follow the treatment by White (1991) and neglect the initial temperature of the infalling flow, since the accretion disk is cool, and the photospheric temperature. We obtain a relation between temperature in the turbulent layer $T_{\text {turb }}$ and velocity $u$ of flow:

$T_{\text {turb }}(u)=\frac{u}{2 c_{\mathrm{p}}}(\Delta v-u)$,

where $\Delta v=v_{\text {Kepler }}-v_{\text {eq }}, u$ is the velocity of the gas relative to the photosphere and $c_{\mathrm{p}}$ has the usual thermodynamic meaning as specific heat at constant pressure. For an ideal gas, Eq. (3) simplifies to $T_{\text {turb }}=2.3 \times 10^{5} \mathrm{~K} \frac{\Delta v^{2}}{\left(100 \mathrm{kms}^{-1}\right)^{2}}\left(\tilde{u}-\tilde{u}^{2}\right)$, with $\tilde{u}=\frac{u}{\Delta v}$, so the maximum temperature is reached where the infalling gas has slowed down by $\frac{\Delta v}{2}$. For $\Delta v \approx 400 \mathrm{~km} \mathrm{~s}^{-1}$, the maximum temperature is $9 \times 10^{5} \mathrm{~K}$ and $2.3 \times 10^{5} \mathrm{~K}$ for half the Keplerian velocity. In the example of TW Hya, in both scenarios the formation temperature of $\mathrm{O}$ VI is reached. Because the line-of-sight is perpendicular to equatorial accretion, the peak of the velocities should appear close to the rest wavelength with a line width comparable to the turbulent velocity. In this scenario, it is clearly difficult to explain the shift of the line centre, but this process may contribute to the emission responsible for a broad component, especially in stars with with only slightly shifted emission such as DF Tau, V4046 Sgr and TWA 5 or in TW Hya during the GHRS and STIS observations. For TWA 5, the scenario furthermore explains how accretion, as supported by a measured $\mathrm{H} \alpha \mathrm{EW}$ of $13.4 \AA$, could proceed. This is in spite of the fact that the $\mathrm{X}$-ray radiation originates in a low-density region, probably a corona, in contrast to that expected in a scenario of magneticallyfunnelled accretion in hot spots. 


\subsection{Red-shifted emission}

All UV observations of TW Hya show consistently red-shifted lines. The only possible explanation are matter flows onto the stellar surface because the far side of the star is blocked from view by the star itself. On the other hand, as argued above, the absence of a double-peaked profile with pre- and post-shock emission implies that accretion spots are unlikely to explain their origin. We therefore now discuss a contribution from the accretion funnels. Three-dimensional MHD simulations of Romanova et al. (2004) give accretion spots with inhomogeneous velocity profiles, where the flow speed is close to free-fall at the spot centre and much slower at the edges. Their simulations use ideal MHD equations and do not take into account the heating by turbulence caused by the difference in velocity within the accretion streams. In general the discussion in Sect. 5.4 applies with velocity differences of the order of half the Keplerian velocity, so significant turbulent heating could arise in the funnels themselves, if turbulence is not suppressed by the strong magnetic fields. Further modelling and clarification of the accretion geometry is required to check the resulting emission measures. Figure 7 shows that the shock, given some turbulent broadening in the post-shock zone, can account for a significant proportion of the observed emission in accordance with our post-shock cooling zone simulations.

Interestingly, in MS stars lines red-shifted by a few $\mathrm{km} \mathrm{s}^{-1}$ are also observed (Ayres \& Linsky 1980; Ayres et al. 1983), so there has to be an emission mechanism unrelated to accretion, usually attributed to downflows in the stellar transition region. This phenomenon may occur in CTTS as well.

\subsection{Other emission origins?}

Emission in the disk surrounding the star may in principle explain broad M-shaped lines, but the temperature at the disk surface is only a few thousand K (Herczeg et al. 2004). Neither C III nor $\mathrm{O}$ VI forms there.

Also, for e.g. TW Hya, where exceptionally broad lines are detected, the projected velocity of the disk at the inner disk edge is only $\approx 20 \mathrm{~km} \mathrm{~s}^{-1}$. We observe no variation in the peak shift between the two observations of TW Hya, so it seems unlikely that it is produced by a single feature moving with the rotating disk.

If the lines originate in an extended chromosphere, from the ratio of equatorial velocity and line width we can estimate the height of the emitting structures and find, using the values for O VI $1032 \AA$ from Table 4, that the emission is placed at the stellar surface for T Tau and at about three stellar radii for RU Lup, V4046 Sgr, GM Aur and TW Hya. This can only explain line shifts if we assume upflowing material for RU Lup and downflows for TW Hya stable over all exposures, which seems unlikely.

\subsection{Variability}

In theoretical simulations accretion, jets and wind generation are highly variable processes (von Rekowski \& Brandenburg 2006). Comparing the fluxes of TW Hya in the multiplet C III $1175 \AA$, in observations acquired using FUSE and HST, we find that the flux decreases by approximately one quarter over three years. In contrast, a flux difference of over a factor of five is measured for RU Lup observations (Herczeg et al. 2005). For TW Hya, there are also FUSE observations separated by several years. Unfortunately, the earlier observations are very short and of low SNR. Comparing the GHRS and STIS observations, separated by 4-8 years, the observed line width and shifts appear to be roughly consistent. We next considered correlations between these observations and FUSE data. Excluding the C III $1175 \AA$ multiplet, for which a line-profile analysis is impossible, there are no lines that are in common between the two sets of data. It is expected however, that lines of Si IV, C IV and N V will originate in emission-line regions for which $\mathrm{C}$ III and $\mathrm{O}$ VI emission is detected. In all cases, the line widths fall in the range 100$300 \mathrm{~km} \mathrm{~s}^{-1}$, and for RU Lup, T Tau and DF Tau the lines are all consistently blueshifted, but in the HST observations to a much lesser extent than in the FUSE data, conversely, the TW Hya lines are more redshifted in the FUSE data. This hints at a strong variability of CTTS, where the dominant UV emission mechanism may change between winds and accretion.

Longer monitoring is required to test if the difference between the objects in our sample is due to different phases of activity and accretion rate or if there are other fundamental differences. This is similar to the situation in X-rays, where several luminosity changes are observed, most likely due to coronal flaring on the time scale of hours (Kastner et al. 2002; Günther et al. 2006), but the evidence is inconclusive and some variability might also be due to time-variable accretion rates.

\section{Summary}

We have presented the hot ion lines originating from C III and $\mathrm{O}$ VI in all available CTTS spectra observed with FUSE. The fitted centroids range from about $-170 \mathrm{~km} \mathrm{~s}^{-1}$ to $+100 \mathrm{~km} \mathrm{~s}^{-1}$ and the shifts of the C III $977 \AA$ line and the O VI $1032 \AA$ and $1038 \AA$ doublet are consistent. Most, if not all, lines are superrotationally broadened. The blue-shifted lines could originate in a stellar outflow, maybe a jet; the red-shifted lines are incompatible with current models of magnetospheric accretion. The presence of boundary layers, winds and extended chromospheres is inconsistent with the observed line profiles.

Furthermore, the absorbing column densities observed in Xrays are incompatible with the optical reddening measured assuming a standard gas-to-dust ratio and interstellar extinction law. This situation holds for the most heavily-veiled objects AA Tau, DF Tau, T Tau, SU Aur and, although for lower absolute values of reddening, RU Lup. In cases where data are available from both UV and X-ray, the corresponding objects demonstrate blue-shifted emission in the FUSE observations. We interpret this as a sign of a dust-free absorber, which may consist of shock-heated jet material. In the ratio between the O VII luminosity and the O VI luminosity, all CTTS display a clear excess of soft X-ray emission in the O VII lines. Together with X-ray results, this illustrates that the signatures of a hot accretion spot are most evident in the temperature range 1-2 MK.

Acknowledgements. We thank G. Herczeg and C. Johns-Krull, the referee, for their competent remarks, especially on the FUSE wavelength calibration. The data presented in this paper were obtained from the Multimission Archive at the Space Telescope Science Institute (MAST). STScI is operated by the Association of Universities for Research in Astronomy, Inc., under NASA contract NAS526555. Support for MAST for non-HST data is provided by the NASA Office of Space Science via grant NAG5-7584 and by other grants and contracts.

CHIANTI is a collaborative project involving the NRL (USA), RAL (UK), MSSL (UK), the Universities of Florence (Italy) and Cambridge (UK), and George Mason University (USA).

H.M.G. acknowledges support from DLR under 50OR0105.

\section{References}

Ake, T. B., Dupree, A. K., Young, P. R., et al. 2000, ApJ, 538, L87 
Alencar, S. H. P., \& Basri, G. 2000, AJ, 119, 1881

Alencar, S. H. P., \& Batalha, C. 2002, ApJ, 571, 378

Ardila, D. R., Basri, G., Walter, F. M., Valenti, J. A., \& Johns-Krull, C. M. 2002a, ApJ, 566, 1100

Ardila, D. R., Basri, G., Walter, F. M., Valenti, J. A., \& Johns-Krull, C. M. 2002b, ApJ, 567, 1013

Argiroffi, C., Maggio, A., \& Peres, G. 2007, A\&A, 465, L5

Argiroffi, C., Maggio, A., Peres, G., Stelzer, B., \& Neuhäuser, R. 2005, A\&A, 439, 1149

Ayres, T. R. 2005, in 13th Cambridge Workshop on Cool Stars, Stellar Systems and the Sun, ed. F. Favata, et al., ESA SP-560, 419

Ayres, T. R., \& Linsky, J. L. 1980, ApJ, 241, 279

Ayres, T. R., Stencel, R. E., Linsky, J. L., et al. 1983, ApJ, 274, 801

Bally, J., Reipurth, B., \& Davis, C. J. 2007, in Protostars and Planets V, ed. B. Reipurth, D. Jewitt, \& K. Keil, 215

Balucinska-Church, M., \& McCammon, D. 1992, ApJ, 400, 699

Basri, G., \& Batalha, C. 1990, ApJ, 363, 654

Bergin, E., Calvet, N., Sitko, M. L., et al. 2004, ApJ, 614, L133

Beristain, G., Edwards, S., \& Kwan, J. 2001, ApJ, 551, 1037

Bertout, C., \& Genova, F. 2006, A\&A, 460, 499

Bloemen, J. B. G. M. 1987, ApJ, 322, 694

Bouvier, J., Bertout, C., Benz, W., \& Mayor, M. 1986, A\&A, 165, 110

Bouvier, J., Chelli, A., Allain, S., et al. 1999, A\&A, 349, 619

Calvet, N., \& Gullbring, E. 1998, ApJ, 509, 802

Cardelli, J. A., Clayton, G. C., \& Mathis, J. S. 1989, ApJ, 345, 245

Chen, W. P., Simon, M., Longmore, A. J., Howell, R. R., \& Benson, J. A. 1990, ApJ, 357, 224

Coffey, D., Bacciotti, F., Woitas, J., Ray, T. P., \& Eislöffel, J. 2004, ApJ, 604, 758

Cohen, M., \& Kuhi, L. V. 1979, ApJS, 41, 743

Dere, K. P., Landi, E., Mason, H. E., Fossi, B. C. M., \& Young, P. R. 1998, in The Scientific Impact of the Goddard High Resolution Spectrograph, ASP Conf. Ser., 143, 390

Dixon, W. V., Sahnow, D. J., Barrett, P. E., et al. 2007, PASP, 119, 527

Dupree, A. K., Brickhouse, N. S., Smith, G. H., \& Strader, J. 2005a, ApJ, 625, L131

Dupree, A. K., Lobel, A., Young, P. R., et al. 2005b, ApJ, 622, 629

Edwards, S., Fischer, W., Hillenbrand, L., \& Kwan, J. 2006, ApJ, 646, 319

Eislöffel, J., \& Mundt, R. 1998, AJ, 115, 1554

Eisner, J. A., Chiang, E. I., \& Hillenbrand, L. A. 2006, ApJ, 637, L133

Errico, L., Lamzin, S. A., \& Vittone, A. A. 2001, A\&A, 377, 557

Feldman, P. D., Sahnow, D. J., Kruk, J. W., Murphy, E. M., \& Moos, H. W. 2001, J. Geophys. Res., 106, 8119

Gómez de Castro, A. I., \& Ferro-Fontán, C. 2005, MNRAS, 362, 569

Gregory, S. G., Jardine, M., Simpson, I., \& Donati, J.-F. 2006, MNRAS, 371, 999

Güdel, M., \& Telleschi, A. 2007, A\&A, 474, L25

Güdel, M., Skinner, S. L., Briggs, K. R., et al. 2005, ApJ, 626, L53

Güdel, M., Skinner, S. L., Mel'Nikov, S. Y., et al. 2007, A\&A, 468, 529

Günther, H. M., \& Schmitt, J. H. M. M. 2007, Mem. Soc. Astron. Ital., 78, 359

Günther, H. M., Schmitt, J. H. M. M., Robrade, J., \& Liefke, C. 2007, A\&A, 466, 1111

Günther, H. M., Liefke, C., Schmitt, J. H. M. M., Robrade, J., \& Ness, J.-U. 2006, A\&A, 459, L29

Hartigan, P., Raymond, J., \& Hartmann, L. 1987, ApJ, 316, 323

Hartmann, L., \& Stauffer, J. R. 1989, AJ, 97, 873

Herczeg, G. J., Linsky, J. L., Valenti, J. A., Johns-Krull, C. M., \& Wood, B. E. 2002, ApJ, 572, 310

Herczeg, G. J., Wood, B. E., Linsky, J. L., Valenti, J. A., \& Johns-Krull, C. M. 2004, ApJ, 607, 369

Herczeg, G. J., Walter, F. M., Linsky, J. L., et al. 2005, AJ, 129, 2777

Herczeg, G. J., Linsky, J. L., Walter, F. M., Gahm, G. F., \& Johns-Krull, C. M. 2006, ApJS, 165, 256

Hueso, R., \& Guillot, T. 2005, A\&A, 442, 703

Johns-Krull, C. M., \& Herczeg, G. J. 2007, ApJ, 655, 345

Joy, A. H. 1949, ApJ, 110, 424

Kastner, J. H., Huenemoerder, D. P., Schulz, N. S., Canizares, C. R., \& Weintraub, D. A. 2002, ApJ, 567, 434

Kenyon, S. J., \& Hartmann, L. 1995, ApJS, 101, 117

Koenigl, A. 1991, ApJ, 370, L39

Koresko, C. D. 2000, ApJ, 531, L147

Lamzin, S. A. 1998, Astron. Rep., 42, 322

Lamzin, S. A. 2003, Astron. Rep., 47, 498

Lamzin, S. A., Bisnovatyi-Kogan, G. S., Errico, L., et al. 1996, A\&A, 306, 877
Lamzin, S. A., Vittone, A. A., \& Errico, L. 2001, Astron. Lett., 27, 313

Lamzin, S. A., Kravtsova, A. S., Romanova, M. M., \& Batalha, C. 2004, Astron. Lett., 30, 413

Landi, E., Del Zanna, G., Young, P. R., et al. 2006, ApJS, 162, 261

Lavalley-Fouquet, C., Cabrit, S., \& Dougados, C. 2000, A\&A, 356, L41

Mamajek, E. E., Meyer, M. R., \& Liebert, J. 2002, AJ, 124, 1670

Matt, S., Goodson, A. P., Winglee, R. M., \& Böhm, K.-H. 2002, ApJ, 574, 232

Mazzotta, P., Mazzitelli, G., Colafrancesco, S., \& Vittorio, N. 1998, A\&AS, 133, 403

McCandliss, S. R. 2003, PASP, 115, 651

Metchev, S. A., Hillenbrand, L. A., \& Meyer, M. R. 2004, ApJ, 600, 435

Mohanty, S., Jayawardhana, R., \& Barrado y Navascués, D. 2003, ApJ, 593, L109

Muzerolle, J., Hartmann, L., \& Calvet, N. 1998, AJ, 116, 2965

Muzerolle, J., Calvet, N., Briceño, C., Hartmann, L., \& Hillenbrand, L. 2000, ApJ, 535, L47

Najita, J. R., Strom, S. E., \& Muzerolle, J. 2007, MNRAS, 378, 369

Ness, J.-U., Güdel, M., Schmitt, J. H. M. M., Audard, M., \& Telleschi, A. 2004, A\&A, 427, 667

Pudritz, R. E., Ouyed, R., Fendt, C., \& Brandenburg, A. 2007, in Protostars and Planets V, ed. B. Reipurth, D. Jewitt, \& K. Keil, 277

Qi, C., Ho, P. T. P., Wilner, D. J., et al. 2004, ApJ, 616, L11

Quast, G. R., Torres, C. A. O., de La Reza, R., da Silva, L., \& Mayor, M. 2000, in IAU Symp., 28P

Redfield, S., Linsky, J. L., Ake, T. B., et al. 2002, ApJ, 581, 626

Robrade, J., \& Schmitt, J. H. M. M. 2006, A\&A, 449, 737

Robrade, J., \& Schmitt, J. H. M. M. 2007, A\&A, 473, 229

Rodmann, J., Henning, T., Chandler, C. J., Mundy, L. G., \& Wilner, D. J. 2006, A\&A, 446, 211

Rodriguez, L. F. 1995, in Rev. Mex.e Astron. Astrofis. Conf. Ser., ed. S. Lizano, \& J. M. Torrelles, 1

Romanova, M. M., Ustyugova, G. V., Koldoba, A. V., \& Lovelace, R. V. E. 2004, ApJ, 610, 920

Saar, S. H., \& Osten, R. A. 1997, MNRAS, 284, 803

Sallmen, S., Johns-Krull, C. M., Welsh, B., \& Griffiths, N. 2000, in BAAS, 1482 Salyk, C., Blake, G. A., Boogert, A. C. A., \& Brown, J. M. 2007, ApJ, 655, L105 Savage, B. D., \& Mathis, J. S. 1979, ARA\&A, 17, 73

Schaefer, G. H., Simon, M., Beck, T. L., Nelan, E., \& Prato, L. 2006, AJ, 132, 2618

Schegerer, A., Wolf, S., Voshchinnikov, N. V., Przygodda, F., \& Kessler-Silacci, J. E. 2006, A\&A, 456, 535

Schmitt, J. H. M. M., \& Robrade, J. 2007, A\&A, 462, L41

Shu, F., Najita, J., Ostriker, E., et al. 1994, ApJ, 429, 781

Simon, M., Dutrey, A., \& Guilloteau, S. 2000, ApJ, 545, 1034

Stelzer, B., \& Schmitt, J. H. M. M. 2004, A\&A, 418, 687

Stempels, H. C., \& Gahm, G. F. 2004, A\&A, 421, 1159

Stempels, H. C., \& Piskunov, N. 2002, A\&A, 391, 595

Stempels, H. C., Gahm, G. F., \& Petrov, P. P. 2007, A\&A, 461, 253

Strom, K. M., Strom, S. E., Edwards, S., Cabrit, S., \& Skrutskie, M. F. 1989, AJ, 97, 1451

Takami, M., Bailey, J., Gledhill, T. M., Chrysostomou, A., \& Hough, J. H. 2001, MNRAS, 323, 177

Telleschi, A., Güdel, M., Briggs, K. R., et al. 2007, A\&A, 468, 541

Torres, G., Guenther, E. W., Marschall, L. A., et al. 2003, AJ, 125, 825

Uchida, K. I., Calvet, N., Hartmann, L., et al. 2004, ApJS, 154, 439

Uchida, Y., \& Shibata, K. 1984, PASJ, 36, 105

Unruh, Y. C., Collier Cameron, A., \& Guenther, E. 1998, MNRAS, 295, 781

Valenti, J. A., Johns-Krull, C. M., \& Linsky, J. L. 2000, ApJS, 129, 399

van Langevelde, H. J., van Dishoeck, E. F., \& Blake, G. A. 1994a, ApJ, 425, L45

van Langevelde, H. J., van Dishoeck, E. F., van der Werf, P. P., \& Blake, G. A. 1994b, A\&A, 287, L25

Vilhu, O., Gustafsson, B., \& Edvardsson, B. 1987, ApJ, 320, 850

von Rekowski, B., \& Brandenburg, A. 2006, Astron. Nachr., 327, 53

Vuong, M. H., Montmerle, T., Grosso, N., et al. 2003, A\&A, 408, 581

Walter, F. M., Herczeg, G., Brown, A., et al. 2003, AJ, 126, 3076

Webb, R. A., Zuckerman, B., Platais, I., et al. 1999, ApJ, 512, L63

Weinberger, A. J., Becklin, E. E., Zuckerman, B., \& Song, I. 2004, AJ, 127, 2246

White, F. M. 1991, Viscous fluid flow (New York: McGraw-Hill)

Wilkinson, E., Harper, G. M., Brown, A., \& Herczeg, G. J. 2002, AJ, 124, 1077

Wood, B. E., Linsky, J. L., \& Ayres, T. R. 1997, ApJ, 478, 745

Zel'Dovich, Y. B., \& Raizer, Y. P. 1967, Physics of shock waves and hightemperature hydrodynamic phenomena (New York: Academic Press), ed. W. D. Hayes, \& R. F. Probstein 